\title{
LA RETÓRICA INSTITUCIONAL DEL BANCO MUNDIAL EN TORNO A LAS POLÍTICAS DE CIENCIA Y TECNOLOGÍA DE LAS UNIVERSIDADES LATINOAMERICANAS.
}

\section{Hacia la búsqueda de autonomía académica en los países de la región}

\author{
Mariano Diodati (UNR-CONICET)* \\ María Graciela Mendíaz (UNR-Red Educación UNESCO-UNITWIN. Arg.)**
}

\begin{abstract}
Resumen
El trabajo plantea la injerencia de la retórica institucional del Banco Mundial -en adelante BM- en la Educación Superior Universitaria de América Latina, en el contexto de la denominada Sociedad del Conocimiento. En tal sentido, se analiza la idea de Universidad que subyace en el aparato discursivo del mencionado organismo multilateral, a través de las políticas recomendadas en los espacios de Ciencia y Tecnología. Si bien esta problemática ha sido objeto de debate desde diversos sectores académicos, en general, se analiza la incidencia del BM en la configuración de las políticas universitarias implementadas en la región desde fines de la década del ochenta hasta los primeros años del nuevo siglo; detectándose, sin embargo, escasos análisis sobre las actuales argumentaciones discursivas del BM.

De la comunidad académica latinoamericana depende que la relación entre el país hegemónico -con acuerdo de nuestras elites y un número no menor de nuestras universidades-, sea revertida, permitiendo en este sentido, afirmar que bajo el neoliberalismo, la estrecha articulación entre la Universidad y el BM, ha sido sólo, para utilizar la terminología de Marx, un "maridaje fugaz".
\end{abstract}

Licenciado y Profesor en Ciencias de la Educación. Facultad de Humanidades y Artes. Universidad Nacional de Rosario. Doctorando en Humanidades y Artes con mención en Ciencias de la Educación. Escuela de Posgrado de la Facultad de Humanidades y Artes. Universidad Nacional de Rosario. Becario Doctoral de CONICET (Tipo II)

* Magister en Ciencia Política y Sociología (FLACSO). Profesora de Historia. Doctoranda del Programa de Posgrado "Ciencia Política". Facultad de Ciencia Política y RR.II. Universidad Nacional de Rosario. 


\title{
Palabras clave
}

Educación superior universitaria - Ciencia y tecnología - Banco mundial - Sociedad del conocimiento - Retórica.

\begin{abstract}
This report set out the impact of the institutional rhetoric of the World Bank on the Latin-American High School, in the context of the so-called Knowledge Society. In that sense, it is analyzed the idea of College which underlies in the discursive system of that multilateral agency, through the recommended policies in Science and Technology fields. Even though this issue has been the object of debate from several academic areas, in general, it is analyzed the incidence of the WB in the configuration of the implemented high school policies in the region since the end of the eighties till the first years of the new century; having been detected, however, limited analysis on the current discursive argument from the WB.

It depends on the Latin-American academic community that the relationship which was imposed from the hegemonic country -with the consent of our elites and a great number of our colleges-, it is possible to assert that under neoliberalism the close articulation between the College and the WB has only been set to use Marx's terminology, a "brief marriage".
\end{abstract}

\section{Key words}

High school education - Science and technology - World bank - Knowledge society Rhetoric.

\section{Introducción}

A fin de problematizar a la Universidad como objeto de reflexión teórica, se selecciona como eje temático central las vinculaciones que se establecen entre las misiones y tareas que se les asignan a las instituciones de Educación Superior Universitaria -ESU- latinoamericanas en el contexto de la denominada Sociedad del Conocimiento; en la que prevalece un tipo de conocimiento: el producido por las ciencias "duras" en respuesta a la demanda del progreso tecnológico (Borón 2002, 2005). Este trabajo se propone explorar la idea de Universidad que subyace en la retórica institucional del Banco Mundial -BMpara exponer de qué manera las instituciones universitarias se encuentran, al inicio del siglo XXI, enfrentadas ante retos que estarían poniendo en tensión su significado y pertinencia en los campos del saber y en aquellos ligados a la producción de conocimiento (Chaïm Perelman 1949, 1952, 1958, 1970; Borón, 2005). Si bien se reconoce que el debate se ha venido realizando desde sectores académicos latinoamericanos, es posible visualizar que la mayor parte de los estudios, analizan en profundidad la incidencia del BM en la configuración de 
las políticas universitarias implementadas desde fines de la década del '80 en la región. Sin embargo, no abundan estudios que reflexionen sobre el actual aparato discursivo del Organismo Multilateral, pues en comparación con tal período, existe una "laguna o vacío de conocimiento" sobre el tema, donde el $\mathrm{BM}$ reconoce, a través de sus últimas publicaciones institucionales, los déficit de sus lineamientos políticos e ideológicos a trasluz de la implementación de los mismos en las políticas educativas.

Interesa asimismo, reflexionar sobre la mirada que los académicos ubicados en vertientes críticas realizan sobre la acción desarrollada por el BM en torno a la Universidad en el marco de la Sociedad del Conocimiento, siguiendo el enfoque teórico de Boaventura de Sousa Santos (2005); a fin de conocer cómo visualizan los nuevos retos que se le impone a la Universidad, y las propuestas acerca de los cambios que requiere realizar la misma frente a estas "nuevas" variables.

El eje central del trabajo radica en problematizar acerca de la noción de autonomía académica que maneja el BM en materia de ESU en un contexto signado por una mayor fluidez de los contactos internacionales de índole académica, política y técnica; finalización de la guerra fría y emergencia de nuevas tecnologías de comunicación, difundiendo modelos y reglas de funcionamiento institucional que influyeron en todos los niveles educativos $\mathrm{y}$, particularmente, en la Universidad (Ascolani, 2003).

Es de destacar que se adhiere al concepto Organismos Multilaterales, el que se prefiere en reemplazo de Organismos de Cooperación Internacional con la intencionalidad de tomar un distanciamiento crítico de la usanza de estas expresiones comúnmente realizadas por entidades financieras multilaterales, tal es el caso del BM; seleccionado para el análisis por resultar la organización que con mayor fuerza y visibilidad viene influenciado, a través de sus racionalidades operativas, en la configuración de las políticas públicas latinoamericanas, y por la mayor difusión de publicaciones documentales y el acceso a las mismas, en términos comparados con otros organismos multilaterales (Murillo, 2006).

Es, con el propósito de arribar a los objetivos propuestos que se tomarán las principales publicaciones documentales del BM, a fin de evidenciar empíricamente los postulados vertidos por la institución crediticia en torno a la noción de autonomía académica, que tuvieran como eje de análisis a los países latinoamericanos en general y, a Argentina, en particular. Entre estas publicaciones, se considera que "Construir Sociedades de Conocimiento. Nuevo desafío para la Educación Superior" constituye el documento fundamental, pues permite visualizar, nítidamente, las observaciones sobre los roles que corresponden a la Educación Superior Universitaria en la Sociedad del Conocimiento.

En la bibliografía especializada se distingue la idea que sostiene que los organismos multilaterales han incidido con notoriedad, en el lapso de las últimas décadas, en la "hechura" de las políticas públicas de los países "en desarrollo", 
como los denominan estas agencias. Tal incidencia no sólo se configura a partir de directrices y lineamientos, sino también, a través del otorgamiento de créditos, que se traducen, para los países prestatarios, en rígidas condicionalidades políticas (Corbalán, 2002).

A fin de ordenar el trabajo, se lo ha dividido en cuatro apartados. Se trata de reflexiones desarrolladas muy brevemente por el espacio que se dispone.

\section{Restricciones financieras y difusión de modelos de funcionamiento institucional: ¿vulnerabilidad de la autonomía universitaria?}

La tarea de abordar en la actualidad la retórica institucional del BM en materia de ESU, obliga a tomar en consideración las notas que han caracterizado la injerencia de este organismo en las Universidades latinoamericanas. Las "reformas" neoliberales en la ESU deben entenderse dentro de un enfoque que contemple las especificidades, en términos de tradiciones y singularidades, que adquiere la Universidad en la realidad de cada país. Sin embargo, y siguiendo las consideraciones de Nosiglia (2007), es posible sostener que las distintas políticas que se aplicaron para la ESU tuvieron como consecuencia el cambio de estilo en la relación gobierno-Educación Superior, que se fundamenta en el diagnóstico de la crisis del modelo universitario vigente, cuya manifestación más evidente sería la pérdida de calidad de las universidades, producto del aumento explosivo de la matrícula y de la imposibilidad de financiamiento incremental por parte del Estado ante la crisis y el ajuste de los años '80. Esta situación marca el agotamiento de un estilo de relación Estado-Universidad descrito como "benevolente" y el inicio de una nueva relación en la cual, el primero de los actores, exige la rendición de cuentas del accionar de las instituciones a cambio del financiamiento estatal, convirtiéndose en un Estado evaluador (Ribeiro Durham, 1990; Brunner, 1993; Winkler, 1994; Del Bello, 1995). La ESU comienza una "nueva época"; se trata de un tiempo portador de un discurso cuyos ejes vertebrales se traducen en notas como calidad, evaluación, eficiencia, productividad, pertinencia y excelencia, y que refleja más que un interés y búsqueda del mejoramiento educativo, el cambio radical en la orientación y las bases sociales que sustentan el proyecto de ESU (Aboites, 2001).

En Argentina, la sanción de la Ley de Educación Superior № 24.521 implicó la consolidación de los mencionados ejes, implementados en forma progresiva por el Poder Ejecutivo Nacional sobre la base de diagnósticos y proyectos sustentados en presupuestos político-ideológicos neoliberales promovidos por organismos multilaterales (Nosiglia, 2007). En la literatura académica especializada, se reconoce que los Organismos Multilaterales, desde fines de los años 80 , tienen propuestas compartidas para enfrentar ciertos problemáticas, que trasuntan un optimismo excesivo sobre el papel transformador de la educación, "llave maestra" para lograr la equidad, el desarrollo y la ciudadanía ampliada. Estas consideraciones se vislumbran con peso específico a través del documen- 
to de CEPAL-UNESCO, del año 1991 denominado "Educación y conocimiento: eje de la transformación productiva con equidad" el cual pasa a ser utilizado en los discursos oficiales, proyectos educativos y sistemas de evaluación. En otras palabras, es posible afirmar que el mismo resulta inspirador de las Reformas Educativas de Latinoamérica. Desde la mirada de autores ubicados en vertientes académicas críticas se vislumbra la manera en que las recomendaciones y sugerencias de estos organismos fueron conformando una matriz a partir de la cual se diseñaron las notas "reformistas" para la ESU latinoamericana. Gran parte de los organismos multilaterales de crédito preocupados por mucho tiempo en temas de base netamente económica, comienzan, finalizando la década del ' 80 , a concebir a la educación en los países en desarrollo como una prioridad para la formación de recursos humanos productivos en pos de mejorar sus niveles de vida y reducir las diferencias con los países centrales (Comboni Salinas, 2001). Asimismo, es preciso resaltar que la preocupación de las agencias multilaterales en temáticas relacionadas con el campo educativo, debe comprenderse dentro de los cambios tácticos y estratégicos operados en sus misiones y funciones centrales. Siguiendo a R. Torres (2001), se produce un desplazamiento de la UNESCO, que fue progresivamente comandado por el BM, en primer orden, y secundado por el BID. En vínculo directo con el $\mathrm{BM}$, las estrategias de acción, tendencias y lineamientos para la reforma de la ESU, se presentan de manera cuasi regular en las diferentes publicaciones analizadas en el periodo comprendido entre fines de la década del ochenta hasta la actualidad.

Otro punto a destacar es que al inicio del año 2000 el BM publicita un estudio sobre la evolución de la Educación Superior en los países en desarrollo, el que concluye sosteniendo que el énfasis puesto en la Educación Primaria y Secundaria habría procedido en perjuicio de la inversión en el nivel universitario, perturbando el tratamiento global de los sistemas educativos nacionales (Banco Mundial, 2000). Para Ascolani (2003) esta permutación en la percepción implica una variación del sendero definido por las recomendaciones aplicadas en los países latinoamericanos en toda la década del '90, cuyo acento recaía en el desarrollo de la educación básica como modo de acción contra la pobreza. Los intelectuales que habían criticado las recomendaciones educativas del BM por estar sometidas a los mandatos teóricos de la economía neoliberal, percibieron esta renovación de la mirada del organismo sobre la cuestión educativa de los países del tercer mundo como un giro en el sentido correcto, en tanto tendía a valorizar la ESU y con ello, la posibilidad de innovación tecnológica alentada por los gobiernos de los propios países a partir de políticas de investigación y mejora real de la calidad de la educación universitaria.

Más allá de las variaciones que pueden encontrarse en la retórica de las organizaciones multilaterales, y por antonomasia del BM, sostienen que para llegar a un determinado cambio respecto de quién debe tener acceso al conoci- 
miento en la universidad y qué debe conocerse en ella, es necesario comenzar planteando las cuestiones ligadas al poder en las instituciones universitarias (Aboites, 1997, 2001). Al respecto, una distinción que merece efectuarse radica en que si bien en el arsenal discursivo del BM estas temáticas ocupan un lugar protagónico, las líneas referidas al financiamiento también se destacan cuando se analiza la retórica institucional del organismo. Son los temas ligados a la dimensión financiera de la ESU, los que permiten introducir la temática acerca del rol que deben desempeñar los Estados en las Universidades. Las directrices que el BM viene imponiendo en éstas, se basan en premisas, tales como: tender a que los gobiernos finalicen con el "subsidio a los ricos", el cual se encuentra implícito en el tipo de financiamiento que prevalece en la ESU; enfrentar la gran diferenciación de instituciones, incluyendo el desarrollo de organizaciones privadas; incentivar a las instituciones públicas para diversificar recursos de financiamiento, abogando por el costo compartido con estudiantes y relaciones financieras con el gobierno; redefinir el rol del gobierno en ESU; introducir políticas explícitamente diseñadas para dar prioridad a los objetivos de calidad y equidad. Sostiene Bentancur (2004) que el escenario que se vislumbra, luego de la aplicación de las directrices neoliberales, se caracteriza por la presencia de instituciones universitarias públicas que compiten entre sí y con otras organizaciones por contratos y estudiantes; universidades que venden conocimientos aplicados y cursos vinculados a las demandas de sus clientes; instituciones que son evaluadas por los gobiernos y que con frecuencia toman recursos adicionales según su acomodamiento a los lineamientos fijados políticamente y a sus resultados; plantel docente que percibe remuneraciones disímiles y variables, aun cuando cuenten con antecedentes académicos similares, y que orientan sus actividades sobre la base de un repertorio de prioridades fijado de manera externa. Siguiendo las consideraciones del autor de referencia, puede sostenerse que uno de los cambios más trascendentales en las universidades latinoamericanas se relaciona con la puesta en funcionamiento de instrumentos de regulación gubernamental, basados en la combinación de tácticas y procedimientos de evaluación, programas de financiamiento extraordinario y exigencias específicas a las instituciones.

Retomando las consideraciones de Nosiglia, el corte economicista de los postulados del BM centra, en gran medida, los problemas del gobierno en las temáticas vinculadas al financiamiento, el que debe seguir no sólo por la contribución de la ESU al desarrollo económico, sino también, porque las imperfecciones del mercado se reducen las posibilidades del acceso a pobres meritorios. No obstante, se recalca que el gobierno debe hacer uso del financiamiento como medio de estímulo para que las instituciones otorguen respuestas a la capacitación nacional y a las necesidades vinculadas a la investigación. Para el BM, la mayor autonomía administrativa de las instituciones tiende, por un lado, a responsabilizar a la Universidad por su desempeño en el uso de los 
fondos públicos, y por otro, al establecimiento de cambios en la estructura de gestión mediante la incorporación de mecanismos contralores sobre el accionar de la comunidad universitaria. No obstante, y haciendo principal hincapié en la autonomía universitaria desde una dimensión eminentemente académica, para el BM un grado elevado de autonomía universitaria dificulta el "buen" funcionamiento de las tareas académicas al interior de las Universidades: la existencia de universidades eficientes en el futuro, depende en gran medida que las mismas dejen de ser autónomas (Aboites, 1997, 2001). En términos del BM (2003), los obstáculos más significativos para el mejoramiento de la eficiencia de la universidad resultan ser: el énfasis en la autonomía universitaria que rechaza las políticas que vienen del gobierno (o de los administradores universitarios).

\section{Retóricas de una organización multilateral en torno a la Educación Superior Universitaria latinoamericana en el marco de la denominada Sociedad del Conocimiento}

Siguiendo las consideraciones de los autores que abordan la temática, un eje problemático relevante, que aporta al debate que se viene desarrollando, se traduce en el siguiente interrogante: ¿Cuáles son las valoraciones y desafíos que el BM le asigna a la Educación Superior Universitaria, en el marco de la globalización, a la misión de conformar la denominada Sociedad del Conocimiento? Para responder a este interrogante se apela a una publicación del BM que data de inicios del año 2002 - "Construir sociedades de conocimiento. Nuevo desafío para la Educación Superior"-, en la cual se aborda la temática en torno a los desafíos que enfrenta la ESU en la Sociedad del Conocimiento. En este documento el BM realiza un diagnóstico sobre la evolución de los sistemas de ESU en el mundo, cuyo principal propósito es la exploración del rol que debe desempeñar la ESU en la tarea de construir entornos de países que propicien mejoras en la capacidad de integración en una economía mundial basada en el conocimiento. De manera transversal, el informe explora la siguiente pregunta problema: ¿De qué manera los países pueden adaptar y diseñar sus sistemas de Educación Superior bajo la finalidad subyacente de enfrentar, de manera "exitosa", la combinación de desafíos tradicionales y emergentes en tal materia?. Como línea temática recurrente, la cual es análoga al modo operante del organismo a lo largo de las últimas décadas, en la publicación se realiza una valoración de tipo analítica sobre las justificaciones vinculadas con la opción de continuar o no con el apoyo del sector público a la ESU. En este sentido, el BM revisa, una vez más, cuál sería el rol apropiado para el Estado en tal materia, en un contexto en el cual se instituye con fuerza 
que los Estados deben apoyar al crecimiento económico orientado por el conocimiento. Conforme avanza el documento, el organismo analiza sus recientes experiencias en apoyo a la ESU, seleccionando aquellas "lecciones" que se traducirían en formas apropiadas para minimizar los impactos políticos negativos, producto de las reformas acaecidas en países centrales y periféricos en el sector universitario a lo largo de la década del ' 90 . A partir de tal selección, promueve recomendaciones estratégicas que podrían convertirse en futuros involucramientos en materia de ESU.

Otra publicación del BM del año 2003 "Ciencia, Tecnología e Innovación en Argentina. Un perfil sobre temas y prácticas", la cual se vincula de manera directa con la construcción de un sistema argentino de innovación competitivo a nivel global, traza un perfil sobre las fortalezas y debilidades del sistema nacional de innovación argentino a fin de identificar políticas eficaces. El BM sostiene que en el país se han establecido instituciones fuertes para el control y apoyo de la Ciencia y Tecnología. Sin embargo, existe un potencial no realizado para adoptar un enfoque coherente en lo que respecta a la investigación y el desarrollo, en particular, para reforzar la asociación del sector público y el privado. Según el BM, el sistema nacional de innovación argentino se caracteriza por la debilidad de los vínculos entre las empresas privadas, las universidades y las instituciones de investigación; argumentando que los pasos fundamentales para la construcción de un sistema innovación competitivo a nivel global radicará en el fortalecimiento de la educación de graduados universitarios, el reforzamiento de la participación privada en Investigación y Desarrollo y la interrelación entre éstos. Por consiguiente, para el BM el capital humano altamente capacitado se torna como un elemento clave en el desarrollo de un sistema de Ciencia, Tecnología e Innovación -CTI- competitivo. El ingenio y la capacidad empresarial forman la columna vertebral de la economía del conocimiento. Como resultado de ello, la mayoría de los esfuerzos para financiar un sistema sólido de CTI y actualizar la infraestructura tecnológica, resultarían inútiles si no se complementaran con capital humano. Se espera por lo tanto, que la educación formal y la actualización de las competencias tengan un mayor efecto sobre el crecimiento que en épocas pasadas. El capital humano se torna particularmente relevante como transmisor de conocimiento tácito y como facilitador de las redes de conocimiento (De Ferranti y otros, citado por el BM, 2003). Para el organismo, la ESU cumple un rol central porque promueve el incremento de la capacidad intelectual, de la cual depende la producción y la posterior utilización del conocimiento, y porque fomenta el desarrollo de prácticas de aprendizaje permanentes necesarias para la actualización de conocimientos y destrezas. No obstante, para el BM cumplimentar el objetivo de ubicar a la ESU en tal lugar, requiere de la capacidad de instituir nuevas instituciones y formas de competencia entre las organizaciones universitarias. El propósito del organismo radica pues, en forzar a las instituciones más tradicionales a 
ajustarse a los nuevos requerimientos tecnológicos para el logro del aprovechamiento de las oportunidades que ofrecen las tecnologías de la información y las comunicaciones.

Entre los cambios vinculados a las necesidades educativas y de capacitación, se distinguen tres actividades que, realizadas por las Universidades, les ayudarían a estructurar sociedades democráticas basadas en el conocimiento: a) apoyo a la innovación mediante la generación de conocimiento nuevo, acceso a depósitos mundiales de conocimiento y adaptación del conocimiento a usos locales; b) contribución a la formación de capital humano mediante la capacitación de una fuerza de laboral calificada y adaptable de alto nivel, incluidos científicos, profesionales, técnicos, maestros de educación básica y secundaria y futuros dirigentes gubernamentales, de la administración pública y de las empresas; y c) contribución a la construcción de la democracia, la nación y la cohesión social.

Otro de los aspectos que merece destacarse se vincula con el posicionamiento que el BM adopta en torno a la educación humanista. Tanto los países industrializados como aquellos en desarrollo necesitan líderes, ciudadanos instruidos y trabajadores capacitados para desempeñarse en la industria, el gobierno, la política y en el mundo académico. La educación humanista permite, potencialmente, promover la responsabilidad ciudadanía, el comportamiento ético, el interés por la educación, el desarrollo profesional en diversos campos, e incluso la integración mundial; al mismo tiempo que fortalece a la sociedad civil, estimula la amplitud de criterio, el pensamiento crítico y la capacidad de comunicación, elementos esenciales para el funcionamiento eficaz de una democracia participativa. Sin embargo, estos argumentos no surgen a partir del documento en análisis, sino que se hacen evidentes, por primera vez, en el año 2003 en una publicación conjunta con la UNESCO -"La Educación Superior en los Países en desarrollo: peligros y promesas"-. En el documento se hace referencia a los obstáculos de la implementación de una educación humanista, que en los países en desarrollo se encuentra con obstáculos de índole económica pero, los más importantes, son de carácter filosófico.

Entonces, según lo manifestado por el BM, surge la imperiosa necesidad de construir un nuevo marco de desarrollo que apoye el crecimiento basado en el conocimiento y la tarea de fomentar la inclusión de sistemas de educación ampliados e incluyentes, a los cuales tengan acceso segmentos más grandes de la población. Se requieren sistemas de ES que impartan habilidades de más alto nivel a una proporción mayor de la fuerza laboral, fomenten el aprendizaje continuo de los ciudadanos, con énfasis en la creatividad y la flexibilidad, para permitir la constante adaptación de las demandas cambiantes de una economía basada en el conocimiento, y promover el reconocimiento internacional de los títulos otorgados por las instituciones educativas del país. De acuerdo a estas consideraciones el BM considera que existen tres grandes vías que contribu- 
yen al fomento del cambio en lo que concierne a necesidades de educación y capacitación: a) más educación para más personas; b) aprendizaje continúo; y c) homologación internacional de títulos.

Asimismo, interesa rescatar el acento colocado por el BM en torno al aprendizaje continuo, por cuanto el discurso del organismo se focaliza principalmente en considerar que el conocimiento, las habilidades y las ocupaciones tienen intrínsecamente una vida útil demasiado corta. Por lo tanto, resalta la necesidad de incentivar los argumentos a favor de la creciente importancia de la educación continuada y de la actualización periódica de las capacidades y calificaciones individuales; pues, en las economías del conocimiento, las nuevas competencias que valoran los empleadores se relacionan con comunicaciones verbales y escritas, trabajo en equipo, enseñanza entre pares, creatividad, capacidad de pensamiento visionario y de ajuste a los cambios, creatividad, recursividad; al respecto resulta interesante destacar que para el organismo muchas de estas competencias implican habilidades sociales, humanas e interculturales que, por lo general, no se enseñan en las disciplinas relacionadas con ciencia o tecnología. Los currículos de la ESU tienden a ser especializados debido a que muchos campos requieren de ciertas habilidades reconocidas, definidas y mensurables. Sin embargo, siempre que sea posible, es primordial enriquecer dichos curriculum con temas generales.

\section{Acerca de algunos enfoques teóricos respecto a la retórica del BM en las últimas décadas}

Por lo expuesto, las libertades académicas aparecen menoscabadas porque las instituciones universitarias tienen que adaptarse a los imperativos que derivan de la lógica mercantil. Asimismo, puede visualizarse cómo frente a esta lógica, ciertas temáticas aparecen como ignoradas, al tiempo que los académicos se tornan cada vez más dependientes de las decisiones de la gestión universitaria. En el actual escenario, la responsabilidad de las universidades aparece con fuerza a la par que la coerción por ser pertinentes frente a las demandas de las sociedades (Borón, 2005).

En palabras de Aboites (2001), proceder a una completa reorientación "empresarial" del proceso educativo superior en las universidades públicas incluye desde la adopción del criterio de "pertinencia" en las carreras y estudios profesionales hasta un cambio radical del "tono" institucional, con la introducción de valores como las competencias, la homogeneidad y la retribución monetaria -merit pay- como elementos claves del mejoramiento académico, lo que se traduce en la competencia entre estudiantes por alcanzar algunas becas o premios, y una definición única y básica del conocimiento indispensable y necesario en cada profesión. En términos generales, y según Bentancur (2004) se intensifica la idea que las universidades deben hacerse cargo de los requerimientos del sector productivo; tendencias que se tornan significativamente peligrosas en 
condiciones estructurantes de restricción financiera que puede llevar a atender prioritariamente el interés de algunos actores externos: los que dispongan de capacidad de compra de los servicios universitarios, en desmedro del resto. Pero, difícilmente éstos, inviertan en programas y planes de investigación y formación que apunten a acumulaciones y resultados a mediano o largo plazo. Por tanto, para el autor de referencia, se reducen los márgenes de las instituciones universitarias para orientar con sus propios criterios sus actividades, pasando a depender de condicionantes externas y cambiantes, y de tendencias de corte instrumental y cortoplacistas. Para Boaventura de Sousa Santos (2007) con mayor notoriedad desde la década de los ' 90 , la universidad, de creadora de condiciones para la competencia y para el éxito en el mercado, se transformó por sí misma, gradualmente, en un objeto de competencia, es decir, en un mercado. Este traspaso del límite en la presión productivista la desvirtúa en su esencia. La mayor autonomía que fue concedida a las universidades no tuvo como objetivo preservar la libertad académica, sino crear condiciones para que se adaptaran a las exigencias de la economía. Para el autor, las ideas que orientan la reforma de la educación propuesta por el BM, y más recientemente para la idea de reconversión de éste en banco del conocimiento, no son en su gran mayoría originarias de los think tanks del BM. La importancia que el banco asume en este campo en los países periféricos y semi-periféricos reside en el modo como sintetiza estas ideas y las transforma en condicionamientos de ayuda para el "desarrollo". Al respecto, señala Borón (2005) que no resulta necesario comunicar que un banco, no importa cómo se autodenomine o cómo quiera aparecer ante la opinión pública, es siempre un banco, y la tendencia natural de todo banquero es considerar a todas las cosas de este mundo, y por cierto a todas las instituciones y prácticas sociales, como simples partidas de una tabla de cálculos de costo-beneficio. En síntesis, la inculcación ideológica se sirve de análisis sistemáticamente sesgados contra la educación pública para demostrar que la educación es potencialmente una mercancía como cualquiera otra y que su conversión en mercancía educativa explica la doble constatación de superioridad del capitalismo en cuanto organizador de las relaciones sociales y de la superioridad de los principios de la economía neoliberal (Santos, 2007).

Según Santos, el celo reformista del Banco dispara en todas las direcciones donde identifica las deficiencias de la universidad pública y, en éstas, uno de los principales objetivos es la posición de poder de los docentes; la libertad académica es vista como un obstáculo a la empresarialización de la universidad y a la responsabilidad de la universidad ante las empresas que pretenden sus servicios. Además, según señala el BM, el poder de una universidad debe descentrarse de los docentes para dirigirse a los administradores entrenados para promover alianzas con agentes privados. 
Siguiendo las consideraciones de Ibarra Colado (2001), la tendencia creciente de comercialización de la universidad se inscribe en esta lógica, modificando las prácticas académicas y reblandeciendo los preceptos éticos del pasado. Y, a través de este nuevo modo de racionalidad, el académico transforma sus prácticas hasta "fundarse" como un homo economicus ilustrado, en un industrial del saber cuya finalidad es producir conocimientos que le posibiliten maximizar tanto sus ingresos monetarios como su capital simbólico. A medida que se modifican los fines y las modalidades que impulsan la producción del conocimiento, y se transforma como mercancía capaz de ser apropiada mediante su intercambio en el mercado, comienza a trastocarse la propia naturaleza, contenido y organización de la profesión académica otorgando lugar a conductas oportunistas basadas en la premisa de actuar siempre en provecho personal, lo que paulatinamente ha provocado la disminución de la fortaleza ética de una organización institucional que se desenvolvió, por largo tiempo, como referente cultural de la sociedad, tal como sostienen Readings (1997) e Ibarra Colado (2001). Para este último, la universidad y sus académicos se mueven motivados más por el dinero que produce el conocimiento que por el conocimiento que contribuye al bienestar general de la sociedad; se trata de la producción de saberes que adquieren su legitimidad en la medida en la que son capaces de traducirse en un valor económico intercambiable en el mercado. Se impone la fórmula a la que refiere Hinkelammert (2007): "un nuevo modelo de desarrollo basado en el capital humano", producto de una estrategia mundial, conducida por el BM, para reducir toda la educación, pero especialmente la universidad, a un lugar de producción de capital humano, que es considerado un medio de producción altamente especializado.

De esta manera, se presenta la antinomia entre autonomía vs. heteronomía. Al respecto, Naishtat (2001), quien basado en el enfoque teórico de Derrida al acuñar la expresión "antinomias de la razón universitaria", define una antinomía como la resultante de un conflicto entre dos proposiciones contradictorias -tesis y antitesis- apoyadas ambas por argumentos. La tesis que defiende la autonomía se encuentra aferrada al argumento que la Universidad moderna es una institución cuyo objeto es la creación y transmisión del conocimiento según una idea de la razón. Esta vocación le da un carácter crítico y reflexivo que deja en plena libertad académica y excluye, en primer lugar, cualquier imposición externa o dogmática en los contenidos de enseñanza y de investigación y, en segundo lugar, cualquier evaluación extra-académica del desempeño universitario (Naisthat, 2001) En contraposición, los argumentos que defienden la heteronomía, se fundamentan en sostener que la autonomía universitaria es una institución autorizada y concedida por el Estado en un marco legal apropiado. La universidad depende en última instancia del marco regulatorio estatal. En el caso de la Universidad pública y/o estatal, esta dependencia se traduce también en dependencia financiera. Por ende la Universidad debe rendir cuentas al Estado; 
la pertinencia social de sus saberes, el cumplimiento apropiado de sus metas son elementos susceptibles de una mirada estatal y de una evaluación externa. Esta dependencia se ve agravada en el caso de las facultades profesionales, donde ha de considerarse la habilitación del título profesional, la evolución del empleo y de los perfiles y prácticas profesionales. Merece destacarse, al interior de esta problemática, la propuesta teórica de Santos (1994) realiza un abordaje analítico en torno a la universidad, en el marco de los países centrales, sobre sus crisis y sus retos hacia finales del siglo XX. El autor, que si bien proviene de un espacio con realidades muy ajenas a las de la región, identifica tres tipos de crisis a las que se enfrentaba la Universidad, a saber: a) crisis de hegemonía la cual se entiende como resultante de las contradicciones entre las funciones tradicionales de la universidad y las que fueron atribuidas durante el siglo XX; tal situación conllevó a que la universidad sea incapaz de desempeñar ambas adecuadamente debido a que por un lado se encontraba la producción de alta cultura, el pensamiento crítico y los conocimientos "ejemplares" destinados a la formación de las élites y, por la otra vertiente, la producción de patrones culturales y conocimientos instrumentales de tipo utilitario para la formación de una mano de obra calificada en pos del desarrollo capitalista. Tal situación conllevó a que tanto el Estado como los agentes económicos busquen por fuera de los márgenes de la universidad medios alternativos conducentes al logro de los mencionados objetivos; lo cual se traduce, en que la universidad deje de ser la única institución en el campo de la ES y en la producción de la investigación; b) crisis de legitimidad por dejar de ser consensual ante la contradicción entre la jerarquización de los saberes especializados -restricciones al ingreso universitario y a la certificación de competencias- y las exigencias sociales y políticas de democratización universitaria, junto con la reivindicación de igualdad de oportunidades; c) crisis institucional producto de la contradicción generada entre la reivindicación de autonomía en la definición de valores y objetivos de la universidad y la presión en aumento para incorporar a la organización institucional en criterios de eficiencia y productividad empresarial o responsabilidad social.

Otra voz que aporta a la problemática que se viene desarrollando es la de López Segrera (2006), para quién la autonomía, que permite a la universidad cumplir en forma cabal sus cometidos, debe ir acompañada de la rendición de cuentas a la sociedad y al Estado (accountability), lo que implica un estilo de rendición social de cuentas que trasciende lo meramente contable; autonomía es la total libertad que los miembros de una universidad tienen para explorar todas las áreas del saber y corrientes del pensamiento humano y para trasmitirlos a sus estudiantes, lo que no significa para el autor, eludir su responsabilidad educativa, ni dejar de rendir cuentas ante la sociedad del uso de los recursos públicos puestos a su disposición para cumplir sus tareas académicas.

En resumen, ¿A qué tipo de exigencias fue enfrentada la Universidad? La Universidad ha sido enfrentada a exigencias contrapuestas con un efecto 
convergente que desestabiliza su institucionalidad actual. Por un lado, la presión hiper-privatizadora de la mercantilización del conocimiento de las empresas concebidas como consumidoras, usuarias y al mismo tiempo como co-productoras del conocimiento científico; una presión que lleva a reducir la responsabilidad social de la universidad y su capacidad de producir conocimiento económicamente útil, es decir, comercializable. Por otro lado, una presión hiper-pública social difusa que despedaza el espacio público de la Universidad en nombre de un espacio público más amplio atravesado por confrontaciones más heterogéneas y por concepciones de responsabilidad social más exigentes (Sousa Santos, 2007). Según Santos (2007) estas presiones han llevado a desestabilizar la institucionalidad de la Universidad, provocando una profunda fractura en la identidad social y cultural.

\section{A modo de cierre: diferentes propuestas desde la mirada de intelectuales y académicos para enfrentar la situación actual}

De acuerdo al enfoque de López Segrera (2006), la crisis institucional de la ESU es posible evitarla al impedir que la Universidad se transforme en un servicio al que se tiene acceso por la vía del consumo y no de la ciudadanía. Esto implica llevar a cabo una reforma en consonancia con un proyecto de nación "aggiornado" que considere a la educación como un bien público. Retomando a Santos, postula que en este marco la Universidad debería convertirse en lo nacional, en productora de equidad y no en reproductora de la desigualdad. A fin de superar esta crisis, debe ser dotada de condiciones idóneas, tanto financieras como institucionales, para el logro de una universidad autorregulada que se encuentre en constante cambio cuando así lo requiera la propia institución; no es el exceso de autonomía lo que impide a las universidades públicas cumplir su compromiso social, sino la falta de medios financieros para desarrollar las nuevas funciones que exige la sociedad del conocimiento (González Casanova, 2001; Sousa, 2004; Borón, 2005; López Segrera, 2006).

Ha podido visualizarse cómo las directrices emanadas del BM desde la última década, exige realizar mayores esfuerzos a las universidades, fundamentalmente a las públicas. La situación se presenta casi como de colapso en muchos países periféricos y se torna difícil en los países semi-periféricos e inclusive en países centrales, aunque en éstos exista mayor capacidad de maniobra para resolver los problemas coyunturales. Asimismo, existen problemas estructurales que son identificables globalmente; la expansión y la transnacionalización del mercado de servicios universitarios de los últimos años han contribuido notoriamente para el aumento de estos problemas, aunque no constituyan la causa única.

Otro aporte interesante que coadyuva a la discusión proviene de Santos (2007) al considerar que al encontrarnos en una fase de transición paradigmática de la ciencia moderna hacia la ciencia posmoderna, la Universidad sólo 
sobrevivirá si asume plenamente esta condición epistemológica. El escenario se enriquece aún más al ser desafiados con propuestas que postulan que a la misma, le compete propiciar la creación de condiciones, a fin de congregar a ciudadanos y a universitarios en auténticas comunidades interpretativas que superen las usuales interacciones, en las cuales los ciudadanos son siempre obligados a renunciar a su propia interpretación de la realidad social. En íntima relación con la tarea de conformar estas comunidades interpretativas, Santos (2007) sostiene que la Universidad no podrá promover su creación en la sociedad si no las sabe conformar en su interior, entre docentes, estudiantes y funcionarios; crear comunidades interpretativas requiere también, someter las barreras disciplinarias y organizativas a una constante presión a través del reconocimiento de múltiples propuestas curriculares puestas en circulación en el interior de las instituciones universitarias. De acuerdo a estas consideraciones resulta necesario que la Universidad reivindique su autonomía y su especificidad organizacional a fin de realizar un diagnóstico de la situación nacional e internacional respecto a la Educación Superior y sus problemas estructurales; así como los que se vinculan a la actualidad pedagógica, didáctica y a las posibilidades de innovación (González Casanova, 2001). Embanderados en la importancia del principio de autonomía institucional debe la Universidad erigirse como una comunidad en constante interpretación y cuestionamiento no sólo de su accionar, sino también de lo que ocurre por fuera de las fronteras institucionales. La acción debe emprenderse rápidamente, pues las "reformas" padecidas por nuestras sociedades en las últimas décadas son, en realidad, acentuados procesos de involución social (Borón, 2003), que estarían poniendo en evidencia lo que Marx ya preanunciara cuando refería a la imposibilidad de articular democracia y capitalismo, relación a la que pertinentemente calificaba como un "maridaje fugaz". De acuerdo a lo expuesto, pareciera confirmarse lo que Borón $(1992,2003)$ afirmara en relación al concepto de reforma al inicio de la década del ' 90 . El politólogo sostiene que dicho concepto ha sufrido una resignificación no menor que dista sustancialmente de la que tuviera en su origen. Hablar de reforma en la tradición del pensamiento político occidental no correspondería a los escenarios actuales sino, muy por el contrario, la expresión contrarreforma es la adecuada pues las políticas llevadas a cabo en la región lejos de tender a procesos reformistas -esto es, cambios graduales en una dirección tendiente hacia una mayor igualdad, bienestar social, y libertad para el conjunto de la población- lo que hicieron fue potenciar una serie de transformaciones que recortaron antiguos derechos ciudadanos, redujeron dramáticamente las prestaciones sociales del Estado y consolidaron una sociedad mucho más injusta y desigual que la que existía al comienzo de la etapa "reformista". Lo que ocurre es que la victoria ideológica del neoliberalismo se expresa, entre otras cosas, por un singular deslizamiento semántico que hace que las palabras pierdan su antiguo significado y adopten otro nuevo. 


\section{Referencias Bibliográficas}

- Aboites, H. (1997) Viento del Norte. TLC y privatización de la Educación Superior en México, México, Universidad Autónoma Metropolitana, Unidad Xochimilco/Plaza y Valdés Editores.

- $\quad$ Aguilar Villanueva, L. (1992) Estudio Introductorio, en Luis F. Aguilar Villanueva (Ed.) El Estudio de las Políticas Públicas, M. Porrúa Grupo Editorial, México.

- Albornoz, M. (2001) Indicadores para la sociedad de la información: una mirada desde Iberoamérica, ponencia presentada en el I Taller Iberoamericano de Indicadores para la Sociedad de la Información; Observatorio das Ciencias e da Tecnología y Ricyt. Lisboa.

- $\quad$ Aristóteles (1999) Retórica, Edición de Antonio Tovar, Centro de Estudios Políticos y Constitucionales, Madrid.

- Ascolani, A. (2003) Política do Banco Mundial para a Educação Latinoamericana, ponencia presentada en Encontro Internacional da Sociedade Brasileira de Educação Comparada: construindo a identidade latino americana, Pontifícia Universidade Católica do Río Grande do Sul/Sociedade Brasileira de Educação Comparada, Porto Alegre, Brasil, publicada en CD, $1^{\text {a }}$ edición, Brasil, ISBN 85-7430-411-5

- Banco Mundial (1995) La enseñanza superior: las lecciones derivadas de la experiencia, en serie "El desarrollo en la Práctica", Washington, D.C.

- Banco Mundial (2000) Educación Superior: Peligros y promesas, Washington D.C.

- Banco Mundial (2005), Ciencia, Tecnología e Innovación en Argentina. Un perfil sobre temas y prácticas. Región de América Latina y el Caribe Departamento de Desarrollo Humano, Washington D.C.

- Bentancur, N. (2004) Gobiernos, Banco Mundial y Universidades: un legado de una década de políticas universitarias en América Latina, en Revista Pensamiento Universitario, № 11.

- Boròn, A. (2000) Tras el búho de Minerva. Mercado contra democracia en el capitalismo de fin de siglo, Epílogo: ¿una teoría social para el siglo XXI?

- Boron, A (2003) Las "reformas del estado" en América Latina: sus negativas consecuencias sobre la inclusión social y la participación democrática, Buenos Aires, Consejo Latinoamericano de Ciencias Sociales.

- $\quad$ Boron, A (2005) Las ciencias sociales en la era neoliberal. Entre la academia y el pensamiento crítico, Conferencia Magistral pronunciada en el XXV Congreso ALAS (Asociación Latinoamericana de Sociología), Porto Alegre, Brasil.

- Brunner, J. J. (1993) Evaluación y financiamiento de la educación superior en América Latina: Bases para un nuevo contrato, en: BALAN (comp.). "Políticas comparadas de educación superior en América Latina”, Santiago de Chile, FLACSO.

- $\quad$ Cazes Menache, D. (2007) A manera de Introducción. Síntesis de reflexiones evocadas en Gandarilla Salgado, José Guadalupe (comp.) "Reestructuración de la Universidad y del conocimiento", Centro de Investigaciones Interdisciplinarias en Ciencias y Humanidades, Coordinación de Humanidades, Universidad Nacional Autónoma de México.

- Cepal-Unesco (1992): Educación y conocimiento: eje de la transformación productiva con equidad. Santiago, agosto.

- Chiroleu, A., Vilosio, L. (2007), Los organismos internacionales y la Educación Superior. Recomendaciones y propuestas para el siglo XXI, en Ideas sobre la cuestión universitaria. Aportes de la RIEPESAL al debate sobre el nuevo marco legal para la Educación Superior, Instituto del Desarrollo Humano, Universidad Nacional de General Sarmiento.

- Comboni Salinas, S. (2001), La Educación Superior vista desde los organismos internacionales y nacionales, ponencia presentada en Red de Investigadores sobre Educa- 
ción Superior/RISEU, UAM-Xochimilco División de Ciencias Sociales y Humanidades, México.

- Coraggio, J. L. (2003) La crisis y las universidades públicas en Argentina, en: Mollis, M. (comp.) Las universidades en América Latina: ¿Reformadas o alteradas?, CLACSO, Bs. As.

- Corbalán, M. (2002) El Banco Mundial. Intervención y Disciplinamiento. El caso argentino, enseñanzas para América Latina, Buenos Aires, Editorial Biblio.

- Del Bello, J. C. (2002), Desafíos De La Política De Educación Superior En América Latina: Reflexiones A Partir Del Caso Argentino con Énfasis Sobre La Evaluación Para El Mejoramiento De La Calidad, Banco Mundial, Washington D.C.

- González Casanova, P. (2001) La Universidad necesaria en el Siglo XXI, México, Ediciones ERA.

- González Casanova, P. (2004) Las nuevas ciencias y las humanidades. De la Academia a la Política (Barcelona: Anthropos), Epílogo: el curso de las ciencias.

- Greco, C. (2004) Epistemología y metodología en la Economía de la Educación Superior, ponencia presentada en IV Encuentro Nacional y II Latinoamericano La Universidad como objeto de investigación, Universidad Nacional de Tucumán.

- Hinkelammert, F. (2007) La Universidad frente a la Globalización en Gandarilla Salgado, José Guadalupe (comp.) "Reestructuración de la Universidad y del conocimiento", Centro de Investigaciones Interdisciplinarias en Ciencias y Humanidades, Coordinación de Humanidades, Universidad Nacional Autónoma de México.

- Ibarra Colado, E. (2005) Origen de la empresarialización de la universidad: el pasado de la gestión de los negocios en el presente del manejo de la universidad, en Revista de la Educación Superior, XXXIV

- Ibarra Colado, E. (2007) Evaluación del trabajo académico y prácticas académicas: impacto de los nuevos modos de regulación, ponencia presentada en Congreso Nacional de Evaluación Educativa, Facultad de Ciencias de la Educación, Universidad Autónoma de Tlaxcala, Tlaxcala, México.

- Levidow, L. (2007) Mercantilizando la Educación Superior: Estrategias neoliberales y contra-estratégicas en Gandarilla Salgado, J. (comp.) "Reestructuración de la Universidad y del conocimiento", Centro de Investigaciones Interdisciplinarias en Ciencias y Humanidades, Coordinación de Humanidades, Universidad Nacional Autónoma de México, México

- López Segrera, F., Abrir, impensar, y redimensionar las ciencias sociales en América Latina y el Caribe. ¿Es posible una ciencia social no eurocéntrica en nuestra región?, en Lander Edgardo (comp.) CLACSO, Consejo Latinoamericano de Ciencias Sociales, Buenos Aires, Argentina.

- Marquina, M, y Soprano, G. (coord.) (2007) Ideas sobre la cuestión universitaria. Aportes de la RIEPESAL al debate sobre el nuevo marco legal para la Educación Superior, Instituto del Desarrollo Humano, Universidad Nacional de General Sarmiento.

- Mendes Pereira, J. M. (2006) El Banco Mundial y la reforma del Estado: ¿más allá del Consenso de Washington? [En línea]. Disponible en http://www.pvp.org.uy/ mendespereira.htmç

- Mollis, M. (comp.) (2003) Las universidades en América Latina: ¿Reformadas o alteradas?, CLACSO, Bs. As.

- Murillo, S. (2006) Banco Mundial: Estado, mercado y sujetos en las nuevas estrategias frente a la cuestión social, Ediciones del Centro Cultural de la Cooperación Floreal, Buenos Aires. 
- Murillo, S. (2007) Naturalización de la pobreza y la desigualdad. Efectos políticos y subjetivos de las estrategias del Banco Mundial, en Revista del Centro Cultural de la Cooperación Floreal Gorini, Año 1, № 1, Buenos Aires.

- Naisthat, F. (2001) Antinomias universitarias y Universitas en la Argentina, en Ciencias Sociales UBA, Buenos Aires.

- Nosiglia, M. C. (2007) Transformaciones en el gobierno de la Educación Superior en la Argentina. Los organismos de coordinación interinstitucional y su impacto en la autonomía institucional, ponencia presentada en el Congreso Latinoamericano de Educación Superior en el Siglo XXI, Buenos Aires.

- Perelman, C y Olbrechsts-Tyteca, L. (1989) Tratado de la Argumentación. La Nueva Retórica, Biblioteca Románica Hispánica, Editorial Gredos, Madrid.

- $\quad$ Readings, Bill (1997) The University in Ruins, Cambridge, Hardvard University Press.

- Ribeiro Durham, E. (1990). Los estudios comparativos de la educación superior en América Latina, en: Kent, R. (comp.). Los temas críticos de la educación superior en América latina. Estudios comparativos, México, Fondo de Cultura Económica.

- Santos, B. (1998) De la mano de Alicia. Lo social y lo político en la posmodernidad, Bogotá, Siglo del Hombre Editores, Ediciones Uniandes, Universidad de los Andes.

- Santos, B. (2007), Tesis para una Universidad pautada por la Ciencia Posmoderna, en Gandarilla Salgado, J. G. (comp.) "Reestructuración de la Universidad y del conocimiento", Centro de Investigaciones Interdisciplinarias en Ciencias y Humanidades, Coordinación de Humanidades, Universidad Nacional Autónoma de México, México

- Torres, R. M. (2001) Cooperación Internacional en Educación en América Latina: ¿Parte de la solución o parte del problema?, [En línea]. Disponible en http://www.oei.es/equidad/ coopal.PDF

- Tussie, D. (1997), El BID, el Banco Mundial y la Sociedad Civil: Nuevas Formas de Financiamiento Internacional, Oficina de Publicaciones del CBC, Universidad de Buenos Aires - FLACSO, Buenos Aires.

- Vilas, C. (2000) ¿"Más allá del "Consenso de Washington"?. Un enfoque desde la política de algunas propuestas del Banco Mundial sobre reforma institucional, [En línea]. Disponible en http://www.clad.org.ve/rev18/vilas.pdf

- Wallerstein, I. (coord.) (2003) Abrir las ciencias sociales. Informe de la Comisión Gulbenkian para la reestructuración de las ciencias sociales. Siglo XXI. México. 5ta. Edición en españolAboites, H (1996) Banco Mundial y Universidad: el fin de la autonomía, el comienzo del nuevo cogobierno. Universidad Autónoma Metropolitana-Xochimilco, ponencia presentada en la Reunión de Análisis de las Ciencias, la Educación y la Cultura, Cuernavaca, Morelos.

- Winkler, R. (1994) La Educación Superior en América Latina. Cuestiones sobre eficiencia y equidad, Serie Documentos para la Discusión del Banco Mundial 77.S, Washington D.C.

- World Bank, (1995) Staff appraisal report, Argentina Higher Education Reform Project, Human Resources Operations Division, Country Departament I, Latin America and the Caribbean Region.

- World Bank (2003) Constructing knowledge societies: new challenges for tertiary education, Washington D.C. 